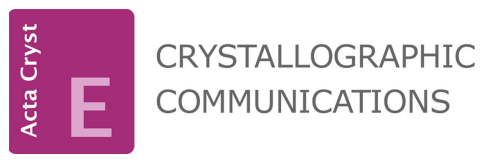

ISSN 2056-9890

Received 22 December 2014

Accepted 13 January 2015

Edited by V. V. Chernyshev, Moscow State University, Russia

Keywords: crystal structure; crotonaldehyde; semicarbazone; thiosemicarbazone; powder X-ray diffraction; supramolecular structure; hydrogen bond; one-dimensional chain; twodimensional networks

CCDC references: 1043290; 1043289 Supporting information: this article has supporting information at journals.iucr.org/e
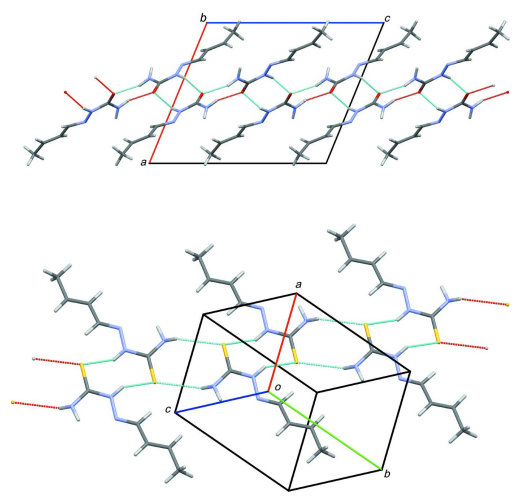

OPEN $\odot$ ACCESS

\section{Crystal structures of crotonaldehyde semicarbazone and crotonaldehyde thiosemicarbazone from X-ray powder diffraction data}

\author{
Atef Arfan and Mwaffak Rukiah* \\ Department of Chemistry, Atomic Energy Commission of Syria (AECS), PO Box 6091, Damascus, Syrian Arab Republic. \\ *Correspondence e-mail: cscientific@aec.org.sy
}

Crotonaldehyde semicarbazone \{systematic name: $(E)-2-[(E)-$ but-2-en-1-ylidene]hydrazinecarboxamide\}, $\mathrm{C}_{5} \mathrm{H}_{9} \mathrm{~N}_{3} \mathrm{O}$, (I), and crotonaldehyde thiosemicarbazone \{systematic name: $(E)-2-[(E)$-but-2-en-1-yldene]hydrazinecarbothioamide\}, $\mathrm{C}_{5} \mathrm{H}_{9} \mathrm{~N}_{3} \mathrm{~S}$, (II), show the same $E$ conformation around the imine $\mathrm{C}=\mathrm{N}$ bond. Compounds (I) and (II) were obtained by the condensation of crotonaldehyde with semicarbazide hydrochloride and thiosemicarbazide, respectively. Each molecule has an intramolecular $\mathrm{N}-\mathrm{H} \cdots \mathrm{N}$ hydrogen bond, which generates an $S(5)$ ring. In (I), the crotonaldehyde fragment is twisted by $2.59(5)^{\circ}$ from the semicarbazide mean plane, while in (II) the corresponding angle (with the thiosemicarbazide mean plane) is $9.12(5)^{\circ}$. The crystal packing is different in the two compounds: in (I) intermolecular $\mathrm{N}-\mathrm{H} \cdots \mathrm{O}$ hydrogen bonds link the molecules into layers parallel to the $b c$ plane, while weak intermolecular $\mathrm{N}-\mathrm{H} \cdots \mathrm{S}$ hydrogen bonds in (II) link the molecules into chains propagating in [110].

\section{Chemical context}

The chemistry of semicarbazones and thiosemicarbazones is especially interesting due to their special role in biological applications such as anti-proliferative, anti-tumoral, anticonvulsant, anti-trypanosomal, herbicidal and biocidal activities (Beraldo et al., 2002; Kasuga et al., 2003; Teixeira et al., 2003; Beraldo \& Gambino, 2004; Mikhaleva et al., 2008; de Oliveira et al., 2008; Alomar et al., 2012; Gan et al., 2014). They are also important intermediates in organic synthesis, mainly for obtaining heterocyclic rings, such as thiazolidones, oxadiazoles, pyrazolidones, and thiadiazoles (Greenbaum et al., 2004; Küçükgüzel et al., 2006). Semicarbazones and thiosemicarbazones have received considerable attention in view of their simplicity of preparation, various complexing abilities and coordination behavior that can be used in analytical applications (Garg \& Jain, 1988; Casas et al., 2000). They are of interest from a supramolecular point of view since they can be functionalized to give different supramolecular arrays.<smiles>C/C=C/C=N/NC(N)=O</smiles>

(I)

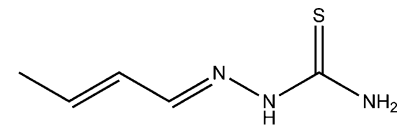

(II)

\section{Structural commentary}

Compounds (I) and (II) crystallize in centrosymmetric space groups $P 2_{1} / c$ and $P \overline{1}$, respectively, with one molecule in the asymmetric unit. Each molecule has an intramolecular $\mathrm{N}-$ $\mathrm{H} \cdots \mathrm{N}$ hydrogen bond (Tables 1 and 2), which forms an $S(5)$ 

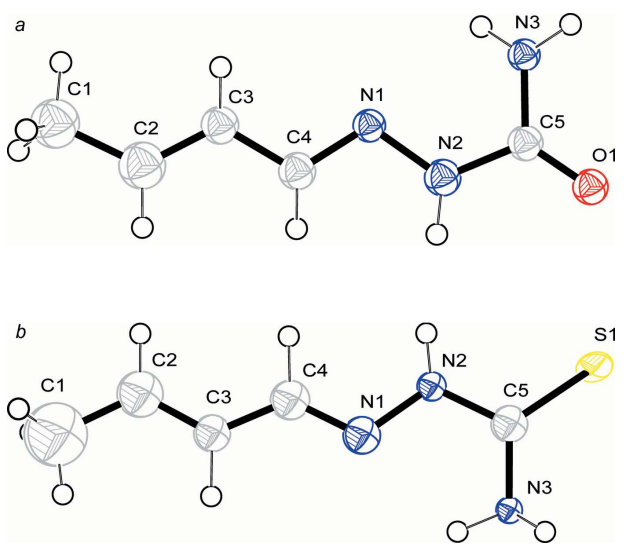

Figure 1

The molecular structures of $(a)$ (I) and (b) (II), showing the atomlabelling schemes. Displacement spheres (and the ellipsoid for S1) are drawn at the $50 \%$ probability level.

ring. The semicarbazone and thiosemicarbazone fragments in the compounds show an $E$ conformation around the imine $\mathrm{C}=\mathrm{N}$ bond. The molecules (Fig. 1) are approximately planar, with a dihedral angle of $2.59(5)^{\circ}$ between the $\mathrm{C} 1 / \mathrm{C} 2 / \mathrm{C} 3$ crotonaldehyde plane and the mean plane of the $C 4 / \mathrm{N} 1 / \mathrm{N} 2 /$ $\mathrm{C} 5 / \mathrm{O} 1 / \mathrm{N} 3$ semicarbazone fragment for (I), and of $9.12(5)^{\circ}$ between the $\mathrm{C} 1 / \mathrm{C} 2 / \mathrm{C} 3$ crotonaldehyde plane and the mean plane of the $\mathrm{C} 4 / \mathrm{N} 1 / \mathrm{N} 2 / \mathrm{C} 5 / \mathrm{S} 1 / \mathrm{N} 3$ thiosemicarbazone fragment for (II). All bond lengths and angles in (I) and (II) are normal and correspond well to those observed in the crystal structures of related semi- and thiosemicarbazone derivatives, viz. acetone semicarbazone and benzaldehydesemicarbazone (Naik \& Palenik, 1974), 3,4- methylenedioxybenzaldehydesemicarbazone (Wang et al., 2004), isatin 3-semicarbazone and 1-methylisatin 3-semicarbazone (Pelosi et al., 2005), 4(methylsulfanyl)benzaldehydethiosemicarbazone (Yathirajan et al., 2006), 4-(methylsulfanyl)benzaldehydesemicarbazone (Sarojini et al., 2007), 5-hydroxy-2-nitrobenzaldehyde thiosemicarbazone (Reddy et al., 2014) and 1-(4-formylbenzylidene) thiosemicarbazone (Carballo et al., 2014).

\section{Supramolecular features}

As a result of the presence of potential hydrogen-donor sites in molecules (I) and (II), supramolecular hydrogen-bonding interactions are observed in both compounds (Tables 1 and 2). In the crystal of (I), molecules are linked by pairs of $\mathrm{N}-$ $\mathrm{H}$. . O hydrogen bonds, forming inversion dimers with $R_{2}^{2}(8)$ ring motifs (Fig. 2a). The resulting dimers are connected through $\mathrm{N}-\mathrm{H} \cdots \mathrm{O}$ hydrogen bonds, forming layers parallel to $b c$ plane. In the crystal of (II), molecules are linked by weak $\mathrm{N}-\mathrm{H} \cdots \mathrm{S}$ hydrogen bonds, forming chains propagating in [110] (Fig. 2b).

\section{Synthesis and crystallization}

All reactions and manipulations were carried out in air with reagent grade solvents. The IR spectra were recorded on a
Table 1

Hydrogen-bond geometry $\left(\AA \AA^{\circ}\right)$ for (I).

\begin{tabular}{lllll}
\hline$D-\mathrm{H} \cdots A$ & $D-\mathrm{H}$ & $\mathrm{H} \cdots A$ & $D \cdots A$ & $D-\mathrm{H} \cdots A$ \\
\hline N3-H2N3 $\cdots \mathrm{N} 1$ & 0.87 & 2.33 & $2.629(19)$ & 100 \\
${\text { N2-H1N2 } \cdots{ }^{\mathrm{i}}}^{\mathrm{i}}$ & 0.88 & 2.07 & $2.910(11)$ & 158 \\
${\text { N3-H1N3 } \cdots \text { O }^{1 i}}$ & 0.91 & 2.04 & $2.914(18)$ & 162 \\
\hline
\end{tabular}

Symmetry codes: (i) $-x+1,-y,-z+1$; (ii) $-x+1, y+\frac{1}{2},-z+\frac{3}{2}$.

Table 2

Hydrogen-bond geometry $\left(\AA,^{\circ}\right)$ for (II).

\begin{tabular}{lllll}
\hline$D-\mathrm{H} \cdots A$ & $D-\mathrm{H}$ & $\mathrm{H} \cdots A$ & $D \cdots A$ & $D-\mathrm{H} \cdots A$ \\
\hline N3-H2N3 $\cdots \mathrm{N} 1$ & 0.89 & 2.17 & $2.641(14)$ & 112 \\
N2-H1N2 S1 $^{\mathrm{i}}$ & 0.86 & 2.83 & $3.473(11)$ & 133 \\
N3-H1N3 $\cdots \mathrm{S} 1^{\mathrm{ii}}$ & 0.87 & 2.77 & $3.398(11)$ & 130 \\
\hline
\end{tabular}

Symmetry codes: (i) $-x+2,-y+1,-z+2$; (ii) $-x+1,-y,-z+2$.

Jasco FT-IR 300E instrument. ${ }^{1} \mathrm{H}$ and ${ }^{13} \mathrm{C}\left\{{ }^{1} \mathrm{H}\right\}$ NMR spectra were recorded on a Bruker Bio spin 400 spectrometer. Microanalysis was performed using EURO EA. Powder X-ray diffraction data were collected with Stoe Transmission diffractometer (Stadi P).

For the synthesis of (I), a mixture of semicarbazide hydrochloride $\left(\mathrm{CH}_{5} \mathrm{~N}_{3} \mathrm{O} \cdot \mathrm{HCl} ; 0.5 \mathrm{~g}, 4.5 \mathrm{mmol}\right)$ and sodium acetate $\left(\mathrm{CH}_{3} \mathrm{COONa} ; 0.75 \mathrm{~g}, 9.1 \mathrm{mmol}\right)$ in $10 \mathrm{ml}$ water was agitated well and crotonaldehyde $(0.5 \mathrm{~g}, 7.1 \mathrm{mmol})$ was added slowly with stirring. On completion of the addition, the reaction mixture was agitated for $24 \mathrm{~h}$ at room temperature. The solid product which formed was separated by filtration and washed with water and finally recrystallized from absolute
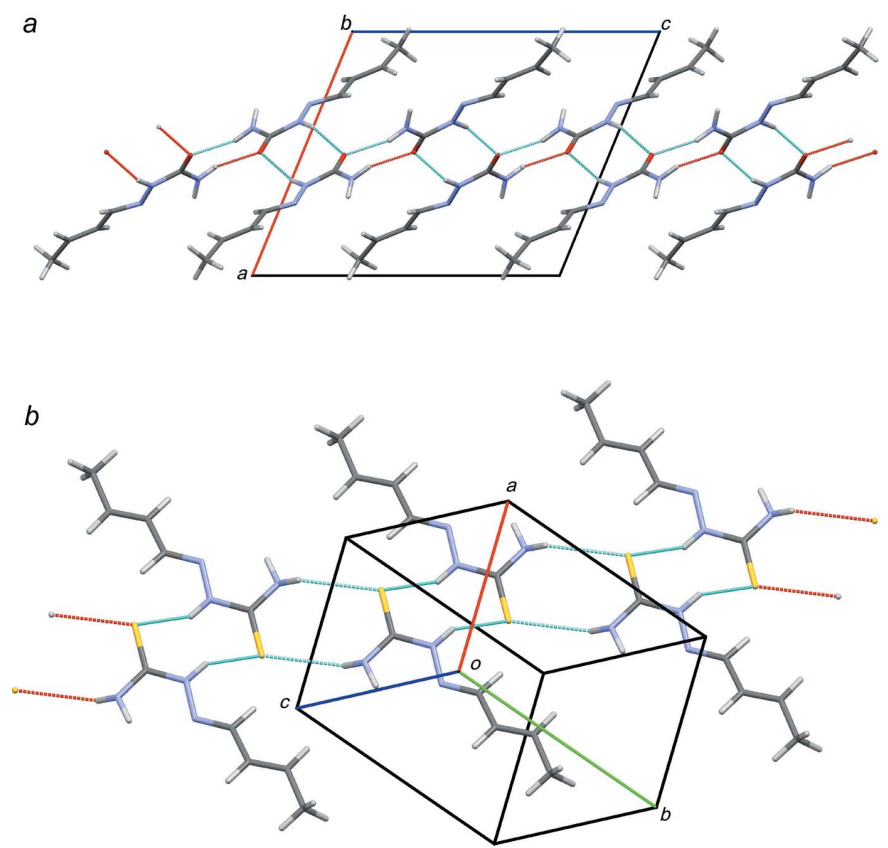

Figure 2

(a) A portion of the crystal packing of (I) viewed down the $b$ axis (parallel to the hydrogen-bonded layer). (b) A portion of the crystal packing of (II), showing the hydrogen-bonded chain of the molecules. Thin dotted lines denote intermolecular hydrogen bonds. 
Table 3

Experimental details.

(I)

Crystal data

Chemical formula

$M_{\mathrm{r}}$

Crystal system, space group

Temperature (K)

$a, b, c(\AA)$

$\alpha, \beta, \gamma\left({ }^{\circ}\right)$

$V\left(\AA^{3}\right)$

$Z$

Radiation type

$\mu\left(\mathrm{mm}^{-1}\right)$

Specimen shape, size (mm)

Data collection

Diffractometer

Specimen mounting

Data collection mode

Scan method

$2 \theta$ values $\left({ }^{\circ}\right)$

Refinement

$R$ factors and goodness of fit

No. of data points

No. of parameters

No. of restraints

$\mathrm{H}$-atom treatment

\author{
$\mathrm{C}_{5} \mathrm{H}_{9} \mathrm{~N}_{3} \mathrm{O}$ \\ 127.15 \\ Monoclinic, $P 2_{1} / c$ \\ 298 \\ 11.1646 (3), 5.13891 (9), 13.0301 (2) \\ 90, $112.3496(11), 90$ \\ $691.43(3)$ \\ 4 \\ Cu $K \alpha_{1}, \lambda=1.5406 \AA$ \\ 0.74 \\ Flat sheet, $8 \times 8$
}

(II)

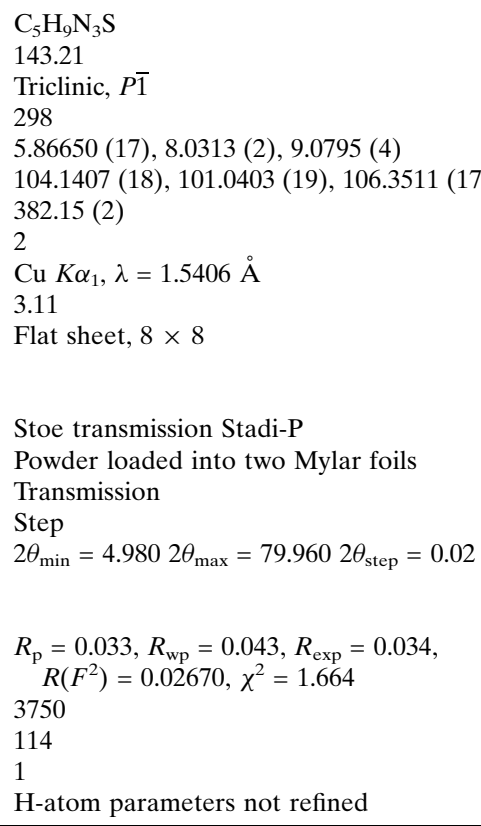

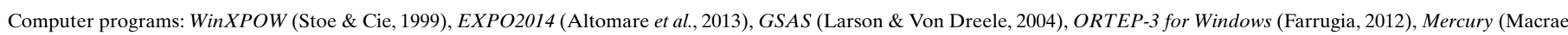
et al., 2006) and publCIF (Westrip, 2010).

ethanol to produce the product (I) (white powder; m.p. 481$482 \mathrm{~K})$ in $55.5 \%$ yield.

IR (KBr, v, cm $\left.{ }^{-1}\right)$ : 3456, 3281, $3192\left(\mathrm{NH}_{2}\right),(1668-1638)$ $(\mathrm{C}=\mathrm{O}) ;{ }^{1} \mathrm{H}$ NMR $\left(400 \mathrm{MHz}, \mathrm{CD}_{3} \mathrm{OD}\right) \delta$ p.p.m. $1.76(d, J=$ $\left.4.42 \mathrm{~Hz}, 3 \mathrm{H},-\mathrm{CH}_{3}\right), 6.43-5.46(m, 2 \mathrm{H},-\mathrm{HC}=\mathrm{CH}-), 7.39(d, J$ $=7.19 \mathrm{~Hz}, 1 \mathrm{H}, \mathrm{HC}=\mathrm{N}-) .{ }^{13} \mathrm{C} \mathrm{NMR}\left(100 \mathrm{MHz}, \mathrm{CD}_{3} \mathrm{OD}\right)$ $\delta$ p.p.m. $18.52\left(\mathrm{CH}_{3}\right), 130.01(-\mathrm{HC}=\mathrm{CH}-), 137.62(-\mathrm{HC}=$ $\mathrm{CH}-), 145.64(\mathrm{~N}=\mathrm{C}), 160.19(\mathrm{C}=\mathrm{O})$. Analysis calculated for (I): C, 47.23; H, 7.13; N, 33.05, 12.58 O\%. Found: C, 46.43; H, $6.08 ; \mathrm{N}, 34.69 \%$

For the synthesis of (II), crotonaldehyde (0.5 g, $7.1 \mathrm{mmol})$ was added to thiosemicarbazide $\left(\mathrm{CH}_{5} \mathrm{~N}_{3} \mathrm{~S} ; 0.65 \mathrm{~g}, 7.1 \mathrm{mmol}\right)$ in $5 \mathrm{ml}$ water and the mixture was stirred at room temperature for $24 \mathrm{~h}$. The product was separated by filtration and recrystallized from absolute ethanol to produce the product (II) (white powder; m.p. 435-436 K) in 72.5\% yield.

IR (KBr, v, cm $\left.{ }^{-1}\right): 3323,3244,3164\left(\mathrm{NH}_{2}\right), 1650(\mathrm{C}=\mathrm{S}) .{ }^{1} \mathrm{H}$ NMR (400 MHz, $\left.\mathrm{CDCl}_{3}\right) \delta$ p.p.m. $1.90(d, J=5.86 \mathrm{~Hz}, 3 \mathrm{H}$, $\left.-\mathrm{CH}_{3}\right), 6.07-6.27(\mathrm{~m}, 2 \mathrm{H},-\mathrm{HC}=\mathrm{CH}-), 6.49(\mathrm{sb}, 1 \mathrm{H}), 7.10(\mathrm{sb}$, 1H) $7.60(d, J=8.57 \mathrm{~Hz}, 1 \mathrm{H}, \mathrm{HC}=\mathrm{N}-), 10.10(s b, 2 \mathrm{H}) .{ }^{13} \mathrm{C}$ NMR (100.6 MHz, $\left.\mathrm{CDCl}_{3}\right) 18.73\left(\mathrm{CH}_{3}\right), 127.70(-\mathrm{HC}=\mathrm{CH}-)$, $140.58(-\mathrm{HC}=\mathrm{CH}-), 146.21(\mathrm{~N}=\mathrm{C}), 177.95(\mathrm{C}=\mathrm{S})$. Analysis calculated for (II): C, 41.93; H, 6.33; N, 29.34.05, $22.39 \mathrm{~S} \%$. Found: C, 41.89; H, 6.25; N, 31.88\%.

\section{Refinement details}

Crystal data, data collection and structure refinement details are summarized in Table 3. Compounds (I) and (II) crystal- lized in the form of a very fine white powder. Since no single crystals of sufficient size and quality could be obtained, the crystal structures of both compounds were determined from X-ray powder diffraction patterns. The powder samples of (I) and (II) were lightly ground in a mortar, loaded into two Mylar foils and fixed onto the sample holder with a mask of suitable internal diameter $(8.0 \mathrm{~mm})$. The powder X-ray diffraction data were collected at room temperature with a STOE transmission STADI-P diffractometer using monochromatic $\mathrm{Cu} K_{a 1}$ radiation $(\lambda=1.54060 \AA)$ selected with an incident beam curved-crystal germanium Ge(111) monochromator with a linear position-sensitive detector (PSD). The patterns were scanned over the angular range 5.0-80.0 $(2 \theta)$. For pattern indexing, the extraction of the peak positions was carried out with the program WinPLOTR (Roisnel \& Rodríguez-Carvajal, 2000). Pattern indexing was performed with the program DICVOL4.0 (Boultif \& Louër, 2004). The first 20 lines of the powder pattern were indexed completely on the basis of a monoclinic cell for (I) and a triclinic cell for (II). The figures of merit (de Wolff et al., 1968; Smith \& Snyder, 1979) are sufficiently acceptable to support the obtained indexing results $[M(20)=50.5, F(20)=71.9(0.0034,83)]$ for $(\mathrm{I})$ and $[M(20)=61.8, F(20)=96.0(0.0051,41)]$ for (II). The best estimated space groups were $P 2{ }_{1} / c$ in the monoclinic system for (I) and $P \overline{1}$ in the triclinic system for (II).

The whole powder diffraction patterns from 5 to $80^{\circ}(2 \theta)$ for the two compounds (I) and (II) were subsequently refined with cell and resolution constraints (Le Bail et al., 1988) using the profile-matching option of the program FULLPROF 

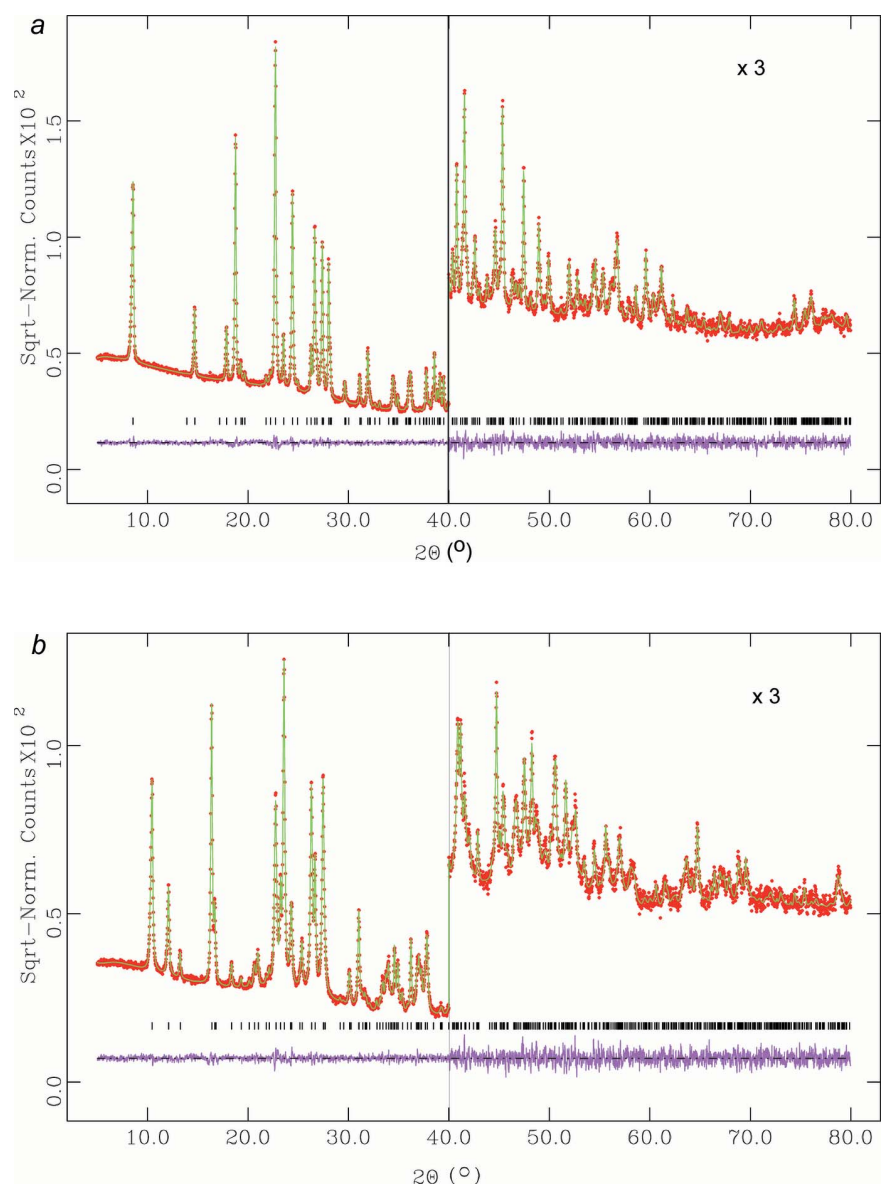

Figure 3

The final Rietveld plots for (a) (I) and (b) (II). Experimental intensities are indicated by dots and the best-fit profile (upper trace) and difference pattern (lower trace) are shown as solid lines. The vertical bars indicate the calculated positions of the Bragg peaks.

(Rodríguez-Carvajal, 2001). The number of molecules per unit cell was estimated to be $Z=4$ for (I) and $Z=2$ for (II). The initial crystal structures for (I) and (II) were determined by direct methods using the program EXPO2014 (Altomare et al., 2013). The models found by this program were introduced into the program GSAS (Larson \& Von Dreele, 2004) implemented in EXPGUI (Toby, 2001) for Rietveld refinement. During the Rietveld refinements, the background was refined using a shifted Chebyshev polynomial with 20 coefficients. The effect of asymmetry of low-order peaks was corrected using a pseudo-Voigt description of the peak shape (Thompson et al., 1987), which allows for angle-dependent asymmetry with axial divergence (Finger et al., 1994) and microstrain broadening, as described by Stephens (1999). The two asymmetry parameters of this function, $S / L$ and $D / L$, were both fixed at 0.022 during this refinement. Intensities were corrected from absorption effects with a function for a plate sample in transmission geometry with a $\mu \cdot d$ value of 0.15 for (I) and 0.72 for (II) ( $\mu$ is the absorption coefficient and $d$ is the sample thickness). These $\mu \cdot d$ values were determined experimentally.

Before the final refinement, all $\mathrm{H}$ atoms were introduced in geometrically calculated positions. The coordinates of these $\mathrm{H}$ atoms were refined with strict restraints on bond lengths and angles until a suitable geometry was obtained, after that they were fixed in the final stage of the refinement. No soft restraints were imposed for (I), while for (II) the $\mathrm{CH}_{3}-\mathrm{CH}$ bond was clearly stretched (close to $1.6 \AA$ ), therefore a single soft restraint was carried out to obtain a normal value (1.49 $\AA$ ). The final refinement cycles were performed using isotropic atomic displacement parameters for the $\mathrm{C}, \mathrm{N}$ and $\mathrm{O}$ atoms, an anisotropic atomic displacement parameter for $\mathrm{S}$ atom in (II) and a fixed global isotropic atomic displacement parameter for the $\mathrm{H}$ atoms. The preferred orientation was modelled with 12 coefficients using a spherical harmonics correction (Von Dreele, 1997) of intensities in the final refinement. The use of the preferred orientation correction leads to a better molecular geometry with better agreement factors. The final Rietveld plots of the X-ray diffraction patterns for both (I) and (II) are given in Fig. 3 .

\section{Acknowledgements}

The authors thank Professor I. Othman, Director General, and Professor Z. Ajji, Head of the Chemistry Department, for their support and encouragement during this work. We also thank Miss D. Naima for her kind assistance.

\section{References}

Alomar, K., Gaumet, V., Allain, M., Bouet, G. \& Landreau, A. (2012). J. Inorg. Biochem. 115, 36-43.

Altomare, A., Cuocci, C., Giacovazzo, C., Moliterni, A., Rizzi, R., Corriero, N. \& Falcicchio, A. (2013). J. Appl. Cryst. 46, 1231-1235.

Beraldo, H. \& Gambinob, D. (2004). Mini Rev. Med. Chem. 4, 31-39.

Beraldo, H., Sinisterra, R. D., Teixeira, L. R., Vieira, R. P. \& Doretto, M. C. (2002). Biochem. Biophys. Res. Commun. 296, 241-246.

Boultif, A. \& Louër, D. (2004). J. Appl. Cryst. 37, 724-731.

Carballo, R., Pino-Cuevas, A. \& Vázquez-López, E. M. (2014). Acta Cryst. E70, o970.

Casas, J. S., Garcl?ía-Tasende, M. S. \& Sordo, J. (2000). Coord. Chem. Rev. 209, 197-261.

Farrugia, L. J. (2012). J. Appl. Cryst. 45, 849-854.

Finger, L. W., Cox, D. E. \& Jephcoat, A. P. (1994). J. Appl. Cryst. 27, 892-900.

Gan, C., Cui, J., Su, S., Lin, Q., Jia, L., Fan, L. \& Huang, Y. (2014). Steroids, 87, 99-107.

Garg, B. S. \& Jain, V. K. (1988). Microchem. J. 38, 144-169.

Greenbaum, D. C., Mackey, Z., Hansell, E., Doyle, P. S., Gut, J., Caffrey, C. R., Lehrman, J., Rosenthal, P. J., McKerrow, J. H. \& Chibale, K. (2004). J. Med. Chem. 47, 3212-3219.

Kasuga, N. C., Sekino, K., Ishikawa, M., Honda, A., Yokoyama, M., Nakano, S., Shimada, N., Koumo, C. \& Nomiya, K. (2003). J. Inorg. Biochem. 96, 298-310.

Küçükgüzel, G., Kocatepe, A., De Clercq, E., Şahin, F. \& Güllüce, M. (2006). Eur. J. Med. Chem. 41, 353-359.

Larson, A. C. \& Von Dreele, R. B. (2004). GSAS. Report LAUR 86748. Los Alamos National Laboratory, New Mexico, USA.

Le Bail, A., Duroy, H. \& Fourquet, J. L. (1988). Mater. Res. Bull. 23, 447-452.

Macrae, C. F., Edgington, P. R., McCabe, P., Pidcock, E., Shields, G. P., Taylor, R., Towler, M. \& van de Streek, J. (2006). J. Appl. Cryst. 39, 453-457.

Mikhaleva, A. I., Ivanov, A. V., Vasil'tsov, A. M., Ushakov, I. A., Ma, J. S. \& Yang, G. (2008). Chem. Heterocycl. Compd, 44, 1117-1122. Naik, D. V. \& Palenik, G. J. (1974). Acta Cryst. B30, 2396-2401. 
Oliveira, R. B. de, de Souza-Fagundes, E. M., Soares, R. P. P., Andrade, A. A., Krettli, A. U. \& Zani, C. L. (2008). Eur. J. Med. Chem. 43, 1983-1988.

Pelosi, G., Pelizzi, C., Belicchi Ferrari, M., Rodríguez-Argüelles, M. C., Vieito, C. \& Sanmartín, J. (2005). Acta Cryst. C61, o5890592.

Reddy, M. S., Sarala, Y., Jagadeesh, M., Das, S. K. \& Ammireddy, V. R. (2014). Acta Cryst. E70, o846.

Rodríguez-Carvajal, J. (2001). Commission on Powder Diffraction (IUCr) Newsletter, 26, 12-19.

Roisnel, T. \& Rodríguez-Carvajal, J. (2000). Mater. Sci. Forum, 378381, 118-123.

Sarojini, B. K., Narayana, B., Bindya, S., Yathirajan, H. S. \& Bolte, M. (2007). Acta Cryst. E63, o2946.
Smith, G. S. \& Snyder, R. L. (1979). J. Appl. Cryst. 12, 60-65.

Stephens, P. W. (1999). J. Appl. Cryst. 32, 281-289.

Stoe \& Cie (1999). WinXPOW. Stoe \& Cie, Darmstadt, Germany.

Teixeira, L. R., Sinisterra, R. D., Vieira, R. P., Doretto, M. C. \& Beraldo, H. (2003). J. Incl. Phenom. Macrocycl. Chem. 47, 77-82.

Thompson, P., Cox, D. E. \& Hastings, J. B. (1987). J. Appl. Cryst. 20, 79-83.

Toby, B. H. (2001). J. Appl. Cryst. 34, 210-213.

Von Dreele, R. B. (1997). J. Appl. Cryst. 30, 517-525.

Wang, J.-L., Jia, Y.-J. \& Yu, M. (2004). Acta Cryst. E60, o662-o663.

Westrip, S. P. (2010). J. Appl. Cryst. 43, 920-925.

Wolff, P. M. de (1968). J. Appl. Cryst. 1, 108-113.

Yathirajan, H. S., Bindya, S., Narayana, B., Sarojini, B. K. \& Bolte, M. (2006). Acta Cryst. E62, o5925-05926. 


\section{supporting information}

Acta Cryst. (2015). E71, 168-172 [doi:10.1107/S2056989015000663]

\section{Crystal structures of crotonaldehyde semicarbazone and crotonaldehyde thio- semicarbazone from X-ray powder diffraction data}

\section{Atef Arfan and Mwaffak Rukiah}

\section{Computing details}

For both compounds, data collection: WinXPOW (Stoe \& Cie, 1999). Data reduction: WinXPOW (Stoe \& Cie, 1999) for (I). For both compounds, program(s) used to solve structure: EXPO2014 (Altomare et al., 2013); program(s) used to refine structure: GSAS (Larson \& Von Dreele, 2004); molecular graphics: ORTEP-3 for Windows (Farrugia, 2012), Mercury (Macrae et al., 2006); software used to prepare material for publication: publCIF (Westrip, 2010).

(I) $(E)-2-[(E)$-But-2-en-1-ylidene]hydrazinecarboxamide

Crystal data

$\mathrm{C}_{5} \mathrm{H}_{9} \mathrm{~N}_{3} \mathrm{O}$

$M_{r}=127.15$

Monoclinic, $P 2{ }_{1} / c$

Hall symbol: -P 2ybc

$a=11.1646(3) \AA$

$b=5.13891(9) \AA$

$c=13.0301(2) \AA$

$\beta=112.3496(11)^{\circ}$

$V=691.43(3) \AA^{3}$

\section{Data collection}

Stoe transmission Stadi-P diffractometer

Radiation source: sealed X-ray tube Ge 111 monochromator
$Z=4$

$F(000)=272$

$D_{\mathrm{x}}=1.222 \mathrm{Mg} \mathrm{m}^{-3}$

$\mathrm{Cu} K \alpha_{1}$ radiation, $\lambda=1.5406 \AA$

$\mu=0.74 \mathrm{~mm}^{-1}$

$T=298 \mathrm{~K}$

Particle morphology: fine powder white

flat sheet, $8 \times 8 \mathrm{~mm}$

Specimen mounting: Powder loaded into two

Mylar foils

Data collection mode: transmission

Scan method: step

$2 \theta_{\text {min }}=5^{\circ}, 2 \theta_{\text {max }}=80^{\circ}, 2 \theta_{\text {step }}=0.02^{\circ}$ 


\section{Refinement}

Least-squares matrix: full

$R_{\mathrm{p}}=0.027$

$R_{\text {wp }}=0.036$

$R_{\text {exp }}=0.029$

$R\left(F^{2}\right)=0.02795$

$\chi^{2}=1.613$

3750 data points
Profile function: CW Profile function number 4 with 21 terms Pseudovoigt profile coefficients as parameterized in (Thompson et al., 1987) Asymmetry correction of Finger et al., 1994. Microstrain broadening by P.W. Stephens, $(1999 . \# 1(\mathrm{GU})=0.000 \# 2(\mathrm{GV})=0.000 \# 3(\mathrm{GW})$ $=10.054 \# 4(\mathrm{GP})=0.000 \# 5(\mathrm{LX})=2.972$ $\# 6($ ptec $)=0.00 \# 7$ (trns) $=0.00 \# 8($ shft $)=$ $0.0000 \# 9(\mathrm{sfec})=0.00 \# 10(\mathrm{~S} / \mathrm{L})=0.0220$ $\# 11(\mathrm{H} / \mathrm{L})=0.0220 \# 12$ (eta) $=0.6000 \# 13(\mathrm{~S} 400$ )$=2.1 \mathrm{E}-01 \# 14(\mathrm{~S} 040)=3.3 \mathrm{E}-01 \# 15(\mathrm{~S} 004)=$ $4.2 \mathrm{E}-02 \# 16(\mathrm{~S} 220)=2.1 \mathrm{E}-02 \# 17(\mathrm{~S} 202)=$ $4.8 \mathrm{E}-03 \# 18(\mathrm{~S} 022)=8.7 \mathrm{E}-02 \# 19(\mathrm{~S} 301)=$ $-3.5 \mathrm{E}-03 \# 20(\mathrm{~S} 103)=5.2 \mathrm{E}-02 \# 21(\mathrm{~S} 121)=$ 5.4E-02 Peak tails are ignored where the intensity is below 0.0010 times the peak Aniso. broadening axis 0.00 .01 .0

121 parameters

0 restraints

$\mathrm{H}$-atom parameters not refined

Weighting scheme based on measured s.u.'s $(\Delta / \sigma)_{\max }=0.03$

Background function: GSAS Background function number 1 with 20 terms. Shifted Chebyshev function of 1st kind 1: 983.4782 : -916.772 3: 421.914 4: -92.3775 5: -9.18321 6: 30.2365 7: -2.25826 8: -10.7421 9: -19.9256 10: 25.6982 11: -24.5216 12: 4.20376 13: 6.93721 14: -3.88406 15: -7.36711 16: 7.71847 17: -1.82508 18: -0.259371 19: -0.220296 20: 0.765767

Fractional atomic coordinates and isotropic or equivalent isotropic displacement parameters $\left(\AA^{2}\right)$

\begin{tabular}{lllll}
\hline & $x$ & $y$ & $z$ & $U_{\text {iso }} / U_{\text {eq }}$ \\
\hline C1 & $0.935(2)$ & $0.8319(18)$ & $0.3282(11)$ & $0.057(5)^{*}$ \\
H1a & 0.88432 & 0.92372 & 0.25964 & $0.075^{*}$ \\
H1b & 0.96701 & 0.95714 & 0.38819 & $0.075^{*}$ \\
H1c & 1.00844 & 0.74948 & 0.31821 & $0.075^{*}$ \\
C2 & $0.855(2)$ & $0.6310(16)$ & $0.3552(13)$ & $0.052(4)^{*}$ \\
H2 & 0.8297 & 0.48475 & 0.30627 & $0.075^{*}$ \\
C3 & $0.8056(15)$ & $0.6448(14)$ & $0.4352(9)$ & $0.033(4)^{*}$ \\
H3 & 0.80211 & 0.8092 & 0.46492 & $0.05^{*}$ \\
C4 & $0.7367(18)$ & $0.4405(17)$ & $0.4558(11)$ & $0.032(4)^{*}$ \\
H4 & 0.73987 & 0.27746 & 0.42622 & $0.075^{*}$ \\
N1 & $0.7027(14)$ & $0.4632(13)$ & $0.5417(7)$ & $0.025(3)^{*}$ \\
N2 & $0.6313(12)$ & $0.2520(15)$ & $0.5530(7)$ & $0.031(3)^{*}$ \\
H1n2 & 0.60928 & 0.12885 & 0.50191 & $0.05^{*}$ \\
C5 & $0.5708(15)$ & $0.252(2)$ & $0.6285(11)$ & $0.029(4)^{*}$ \\
O1 & $0.5060(12)$ & $0.0728(12)$ & $0.6388(6)$ & $0.029(3)^{*}$ \\
N3 & $0.6041(15)$ & $0.4651(13)$ & $0.6956(9)$ & $0.024(3)^{*}$
\end{tabular}




\begin{tabular}{lllll}
$\mathrm{H} 1 \mathrm{n} 3$ & 0.55477 & 0.51321 & 0.73425 & $0.05^{*}$ \\
$\mathrm{H} 2 \mathrm{n} 3$ & 0.67532 & 0.55182 & 0.70648 & $0.05^{*}$ \\
\hline
\end{tabular}

Geometric parameters $\left(\AA,{ }^{\circ}\right)$

\begin{tabular}{llll}
\hline $\mathrm{C} 1-\mathrm{C} 2$ & $1.493(17)$ & $\mathrm{C} 4-\mathrm{H} 4$ & 0.928 \\
$\mathrm{C} 1-\mathrm{H} 1 \mathrm{a}$ & 0.978 & $\mathrm{~N} 1-\mathrm{N} 2$ & $1.387(11)$ \\
$\mathrm{C} 1-\mathrm{H} 1 \mathrm{~b}$ & 0.970 & $\mathrm{~N} 2-\mathrm{H} 1 \mathrm{n} 2$ & 0.883 \\
$\mathrm{C} 1-\mathrm{H} 1 \mathrm{c}$ & 0.975 & $\mathrm{~N} 2-\mathrm{C} 5$ & $1.390(10)$ \\
$\mathrm{C} 2-\mathrm{C} 3$ & $1.353(11)$ & $\mathrm{C} 5-\mathrm{O} 1$ & $1.210(11)$ \\
$\mathrm{C} 2-\mathrm{H} 2$ & 0.956 & $\mathrm{C} 5-\mathrm{N} 3$ & $1.361(11)$ \\
$\mathrm{C} 3-\mathrm{C} 4$ & $1.387(13)$ & $\mathrm{N} 3-\mathrm{H} 1 \mathrm{n} 3$ & 0.911 \\
$\mathrm{C} 3-\mathrm{H} 3$ & 0.936 & $\mathrm{~N} 3-\mathrm{H} 2 \mathrm{n} 3$ & 0.875 \\
$\mathrm{C} 4-\mathrm{N} 1$ & $1.317(11)$ & & \\
& & & \\
$\mathrm{H} 1 \mathrm{a}-\mathrm{C} 1-\mathrm{H} 1 \mathrm{~b}$ & 108.9 & $\mathrm{C} 3-\mathrm{C} 4-\mathrm{N} 1$ & $117.2(12)$ \\
$\mathrm{H} 1 \mathrm{a}-\mathrm{C} 1-\mathrm{H} 1 \mathrm{c}$ & 108.1 & $\mathrm{H} 4-\mathrm{C} 4-\mathrm{N} 1$ & 120.1 \\
$\mathrm{H} 1 \mathrm{a}-\mathrm{C} 1-\mathrm{C} 2$ & 111.0 & $\mathrm{C} 4-\mathrm{N} 1-\mathrm{N} 2$ & $112.5(9)$ \\
$\mathrm{H} 1 \mathrm{~b}-\mathrm{C} 1-\mathrm{H} 1 \mathrm{c}$ & 109.0 & $\mathrm{~N} 1-\mathrm{N} 2-\mathrm{H} 1 \mathrm{C} 2$ & 119.2 \\
$\mathrm{H} 1 \mathrm{~b}-\mathrm{C} 1-\mathrm{C} 2$ & 109.9 & $\mathrm{H} 1 \mathrm{n} 2-\mathrm{N} 2-\mathrm{C} 5$ & $121.9(9)$ \\
$\mathrm{H} 1 \mathrm{c}-\mathrm{C} 1-\mathrm{C} 2$ & 109.9 & $\mathrm{~N} 2-\mathrm{C} 5-\mathrm{O} 1$ & 117.6 \\
$\mathrm{C} 1-\mathrm{C} 2-\mathrm{H} 2$ & 115.9 & $\mathrm{~N} 2-\mathrm{C} 5-\mathrm{N} 3$ & $123.3(11)$ \\
$\mathrm{C} 1-\mathrm{C} 2-\mathrm{C} 3$ & $126.9(9)$ & $\mathrm{O} 1-\mathrm{C} 5-\mathrm{N} 3$ & $124.8(11)$ \\
$\mathrm{H} 2-\mathrm{C} 2-\mathrm{C} 3$ & 116.9 & $\mathrm{C} 5-\mathrm{N} 3-\mathrm{H} 1 \mathrm{n} 3$ \\
$\mathrm{C} 2-\mathrm{C} 3-\mathrm{H} 3$ & 117.3 & $\mathrm{C} 5-\mathrm{N} 3-\mathrm{H} 2 \mathrm{n} 3$ & 119.9 \\
$\mathrm{C} 2-\mathrm{C} 3-\mathrm{C} 4$ & $121.8(10)$ & $\mathrm{H} 1 \mathrm{n} 3-\mathrm{N} 3-\mathrm{H} 2 \mathrm{n} 3$ & 121.6 \\
$\mathrm{H} 3-\mathrm{C} 3-\mathrm{C} 4$ & 119.4 & & 118.4 \\
$\mathrm{C} 3-\mathrm{C} 4-\mathrm{H} 4$ & 119.5 & $\mathrm{~N} 1-\mathrm{N} 2-\mathrm{C} 5-\mathrm{N} 3$ & \\
& & $\mathrm{C} 1-\mathrm{C} 2-\mathrm{C} 3-\mathrm{C} 4$ & \\
$\mathrm{C} 4-\mathrm{N} 1-\mathrm{N} 2-\mathrm{C} 5$ & $-171.0(13)$ & & $-7.4(18)$ \\
$\mathrm{N} 2-\mathrm{N} 1-\mathrm{C} 4-\mathrm{C} 3$ & $178.3(13)$ & & $-177.3(16)$ \\
$\mathrm{N} 1-\mathrm{N} 2-\mathrm{C} 5-\mathrm{O} 1$ & $178.6(13)$ & & $174.0(15)$ \\
& & & \\
\hline
\end{tabular}

Hydrogen-bond geometry $\left(A,{ }^{\circ}\right)$

\begin{tabular}{lllll}
\hline$D-\mathrm{H} \cdots A$ & $D-\mathrm{H}$ & $\mathrm{H} \cdots A$ & $D \cdots A$ & $D-\mathrm{H} \cdots A$ \\
\hline $\mathrm{N} 3-\mathrm{H} 2 \mathrm{~N} 3 \cdots \mathrm{N} 1$ & 0.87 & 2.33 & $2.629(19)$ & 100 \\
$\mathrm{~N} 2-\mathrm{H} 1 \mathrm{~N} 2 \cdots \mathrm{O} 1^{\mathrm{i}}$ & 0.88 & 2.07 & $2.910(11)$ & 158 \\
$\mathrm{~N} 3-\mathrm{H} 1 \mathrm{~N} 3 \cdots \mathrm{O} 1^{\mathrm{ii}}$ & 0.91 & 2.04 & $2.914(18)$ & 162
\end{tabular}

Symmetry codes: (i) $-x+1,-y,-z+1$; (ii) $-x+1, y+1 / 2,-z+3 / 2$.

\section{(II) $(E)-2-[(E)$-But-2-en-1-yldene]hydrazinecarbothioamide}

Crystal data

$\begin{array}{ll}\mathrm{C}_{5} \mathrm{H}_{9} \mathrm{~N}_{3} \mathrm{~S} & b=8.0313(2) \AA \\ M_{r}=143.21 & c=9.0795(4) \AA \\ \text { Triclinic, } P 1 & \alpha=104.1407(18)^{\circ} \\ \text { Hall symbol: }-\mathrm{P} 1 & \beta=101.0403(19)^{\circ} \\ a=5.86650(17) \AA & \gamma=106.3511(17)^{\circ}\end{array}$


$V=382.15(2) \AA^{3}$

$Z=2$

$F(000)=152$

$D_{\mathrm{x}}=1.245 \mathrm{Mg} \mathrm{m}^{-3}$

$\mathrm{Cu} K \alpha_{1}$ radiation, $\lambda=1.5406 \AA$

Data collection

Stoe transmission Stadi-P diffractometer

Radiation source: sealed X-ray tube

Ge 111 monochromator

Refinement

Least-squares matrix: full

$R_{\mathrm{p}}=0.033$

$R_{\text {wp }}=0.043$

$R_{\text {exp }}=0.034$

$R\left(F^{2}\right)=0.02670$

$\chi^{2}=1.664$

3750 data points $\mu=3.11 \mathrm{~mm}^{-1}$

$T=298 \mathrm{~K}$

Particle morphology: fine powder white

flat sheet, $8 \times 8 \mathrm{~mm}$

Specimen mounting: Powder loaded into two Mylar foils

Data collection mode: transmission

Scan method: step

$2 \theta_{\min }=4.980^{\circ}, 2 \theta_{\max }=79.960^{\circ}, 2 \theta_{\text {step }}=0.02^{\circ}$

Profile function: CW Profile function number 4 with 21 terms Pseudovoigt profile coefficients as parameterized in (Thompson et al., 1987) Asymmetry correction of Finger et al., 1994. $\# 1(\mathrm{GU})=0.000 \# 2(\mathrm{GV})=0.000 \# 3(\mathrm{GW})=$ $2.793 \# 4(\mathrm{GP})=0.000 \# 5(\mathrm{LX})=5.477 \# 6(\mathrm{ptec})$ $=2.45 \# 7($ trns $)=0.00 \# 8(\mathrm{shft})=0.0000$ $\# 9(\mathrm{sfec})=0.00 \# 10(\mathrm{~S} / \mathrm{L})=0.0220 \# 11(\mathrm{H} / \mathrm{L})=$ $0.0220 \# 12(\mathrm{eta})=0.6000$ Peak tails are ignored where the intensity is below 0.0010 times the peak Aniso. broadening axis 0.00 .01 .0

114 parameters

1 restraint

$\mathrm{H}$-atom parameters not refined

$(\Delta / \sigma)_{\max }=0.03$

Background function: GSAS Background function number 1 with 20 terms. Shifted Chebyshev function of 1st kind 1: 590.360 2: -469.557 3: $198.1264:-45.25865:-2.756246$ : 13.8508 7: 4.35563 8: -5.95029 9: -12.881510 : 35.6051 11: -12.9276 12: -11.1488 13: 8.85293 14: -2.01034 15: -0.496121 16: 8.3961617 :

-2.33367 18: -5.14527 19: 10.5079 20: -3.85249

Fractional atomic coordinates and isotropic or equivalent isotropic displacement parameters $\left(\AA^{2}\right)$

\begin{tabular}{lllll}
\hline & $x$ & $y$ & $z$ & $U_{\text {iso }} * U_{\text {eq }}$ \\
\hline C1 & $0.184(2)$ & $0.841(2)$ & $0.515(2)$ & $0.103(6)^{*}$ \\
H1A & 0.15342 & 0.79934 & 0.39748 & $0.12^{*}$ \\
H1B & 0.2323 & 0.97016 & 0.55142 & $0.12^{*}$ \\
H1C & 0.02574 & 0.78525 & 0.53491 & $0.12^{*}$ \\
C2 & $0.370(2)$ & $0.7688(17)$ & $0.5865(18)$ & $0.054(5)^{*}$ \\
H2 & 0.53963 & 0.83335 & 0.59455 & $0.055^{*}$ \\
C3 & $0.325(2)$ & $0.6393(16)$ & $0.6524(15)$ & $0.034(5)^{*}$ \\
H3 & 0.14582 & 0.56255 & 0.63049 & $0.055^{*}$ \\
C4 & $0.487(3)$ & $0.5747(19)$ & $0.7264(19)$ & $0.039(5)^{*}$ \\
H4 & 0.66632 & 0.65671 & 0.74816 & $0.055^{*}$ \\
N1 & $0.4514(17)$ & $0.4461(12)$ & $0.7878(15)$ & $0.035(4)^{*}$ \\
N2 & $0.6486(16)$ & $0.4005(12)$ & $0.8462(13)$ & $0.021(4)^{*}$
\end{tabular}




\begin{tabular}{lllll}
$\mathrm{H} 1 \mathrm{n} 2$ & 0.79218 & 0.45838 & 0.841 & $0.05^{*}$ \\
$\mathrm{C} 5$ & $0.611(3)$ & $0.2572(16)$ & $0.907(2)$ & $0.034(4)^{*}$ \\
$\mathrm{~N} 3$ & $0.3681(15)$ & $0.1560(12)$ & $0.8849(13)$ & $0.017(4)^{*}$ \\
$\mathrm{H} 1 \mathrm{n} 3$ & 0.34725 & 0.13246 & 0.97116 & $0.05^{*}$ \\
$\mathrm{H} 2 \mathrm{n} 3$ & 0.26401 & 0.20773 & 0.84645 & $0.05^{*}$ \\
S1 & $0.8446(6)$ & $0.1980(5)$ & $0.9772(6)$ & 0.04081 \\
\hline
\end{tabular}

Atomic displacement parameters $\left(\AA^{2}\right)$

\begin{tabular}{lllllll}
\hline & $U^{11}$ & $U^{22}$ & $U^{33}$ & $U^{12}$ & $U^{13}$ & $U^{23}$ \\
\hline S1 & $0.032(4)$ & $0.039(5)$ & $0.082(8)$ & $0.026(4)$ & $0.043(5)$ & $0.035(5)$ \\
\hline
\end{tabular}

Geometric parameters $\left(\AA,{ }^{\circ}\right)$

\begin{tabular}{|c|c|c|c|}
\hline $\mathrm{C} 1-\mathrm{H} 1 \mathrm{~A}$ & 0.999 & $\mathrm{C} 4-\mathrm{N} 1$ & $1.274(12)$ \\
\hline $\mathrm{C} 1-\mathrm{H} 1 \mathrm{~B}$ & 0.946 & $\mathrm{~N} 1-\mathrm{N} 2$ & $1.361(10)$ \\
\hline $\mathrm{C} 1-\mathrm{H} 1 \mathrm{C}$ & 0.983 & $\mathrm{~N} 2-\mathrm{H} 1 \mathrm{n} 2$ & 0.856 \\
\hline $\mathrm{C} 1-\mathrm{C} 2$ & $1.49(2)$ & $\mathrm{N} 2-\mathrm{C} 5$ & $1.377(13)$ \\
\hline $\mathrm{C} 2-\mathrm{H} 2$ & 0.963 & $\mathrm{C} 5-\mathrm{N} 3$ & $1.376(13)$ \\
\hline $\mathrm{C} 2-\mathrm{C} 3$ & $1.311(13)$ & $\mathrm{C} 5-\mathrm{S} 1$ & $1.638(13)$ \\
\hline $\mathrm{C} 3-\mathrm{H} 3$ & 1.008 & $\mathrm{~N} 3-\mathrm{C} 5$ & $1.376(13)$ \\
\hline $\mathrm{C} 3-\mathrm{C} 4$ & $1.352(14)$ & $\mathrm{N} 3-\mathrm{H} 1 \mathrm{n} 3$ & 0.872 \\
\hline $\mathrm{C} 4-\mathrm{H} 4$ & 1.024 & $\mathrm{~N} 3-\mathrm{H} 2 \mathrm{n} 3$ & 0.894 \\
\hline $\mathrm{H} 1 \mathrm{~A}-\mathrm{C} 1-\mathrm{H} 1 \mathrm{~B}$ & 109.0 & $\mathrm{C} 3-\mathrm{C} 4-\mathrm{N} 1$ & $130.8(16)$ \\
\hline $\mathrm{H} 1 \mathrm{~A}-\mathrm{C} 1-\mathrm{H} 1 \mathrm{C}$ & 106.2 & $\mathrm{H} 4-\mathrm{C} 4-\mathrm{N} 1$ & 116.8 \\
\hline $\mathrm{H} 1 \mathrm{~A}-\mathrm{C} 1-\mathrm{C} 2$ & 108.5 & $\mathrm{C} 4-\mathrm{N} 1-\mathrm{N} 2$ & $118.6(11)$ \\
\hline $\mathrm{H} 1 \mathrm{~B}-\mathrm{C} 1-\mathrm{H} 1 \mathrm{C}$ & 110.2 & $\mathrm{~N} 1-\mathrm{N} 2-\mathrm{H} 1 \mathrm{n} 2$ & 119.6 \\
\hline $\mathrm{H} 1 \mathrm{~B}-\mathrm{C} 1-\mathrm{C} 2$ & 113.8 & $\mathrm{~N} 1-\mathrm{N} 2-\mathrm{C} 5$ & $119.2(10)$ \\
\hline $\mathrm{H} 1 \mathrm{C}-\mathrm{C} 1-\mathrm{C} 2$ & 108.8 & $\mathrm{H} 1 \mathrm{n} 2-\mathrm{N} 2-\mathrm{C} 5$ & 121.2 \\
\hline $\mathrm{C} 1-\mathrm{C} 2-\mathrm{H} 2$ & 115.8 & $\mathrm{~N} 2-\mathrm{C} 5-\mathrm{N} 3$ & $115.6(12)$ \\
\hline $\mathrm{C} 1-\mathrm{C} 2-\mathrm{C} 3$ & $125.6(13)$ & $\mathrm{N} 2-\mathrm{C} 5-\mathrm{S} 1$ & $120.4(11)$ \\
\hline $\mathrm{H} 2-\mathrm{C} 2-\mathrm{C} 3$ & 118.2 & $\mathrm{~N} 3-\mathrm{C} 5-\mathrm{S} 1$ & $123.5(9)$ \\
\hline $\mathrm{C} 2-\mathrm{C} 3-\mathrm{H} 3$ & 116.8 & $\mathrm{C} 5-\mathrm{N} 3-\mathrm{H} 1 \mathrm{n} 3$ & 110.5 \\
\hline $\mathrm{C} 2-\mathrm{C} 3-\mathrm{C} 4$ & $128.4(15)$ & $\mathrm{C} 5-\mathrm{N} 3-\mathrm{H} 2 \mathrm{n} 3$ & 112.2 \\
\hline $\mathrm{H} 3-\mathrm{C} 3-\mathrm{C} 4$ & 113.9 & $\mathrm{H} 1 \mathrm{n} 3-\mathrm{N} 3-\mathrm{H} 2 \mathrm{n} 3$ & 113.2 \\
\hline $\mathrm{C} 3-\mathrm{C} 4-\mathrm{H} 4$ & 111.8 & & \\
\hline $\mathrm{C} 4-\mathrm{N} 1-\mathrm{N} 2-\mathrm{C} 5$ & $-177.4(14)$ & $\mathrm{N} 1-\mathrm{N} 2-\mathrm{C} 5-\mathrm{N} 3$ & $8.0(19)$ \\
\hline $\mathrm{N} 2-\mathrm{N} 1-\mathrm{C} 4-\mathrm{C} 3$ & $175.6(15)$ & $\mathrm{C} 1-\mathrm{C} 2-\mathrm{C} 3-\mathrm{C} 4$ & $-176.2(15)$ \\
\hline $\mathrm{N} 1-\mathrm{N} 2-\mathrm{C} 5-\mathrm{S} 1$ & $179.6(11)$ & $\mathrm{C} 2-\mathrm{C} 3-\mathrm{C} 4-\mathrm{N} 1$ & $-177.6(16)$ \\
\hline
\end{tabular}

Hydrogen-bond geometry $\left(\AA,{ }^{\circ}\right)$

\begin{tabular}{lllll}
\hline$D-\mathrm{H} \cdots A$ & $D-\mathrm{H}$ & $\mathrm{H} \cdots A$ & $D \cdots A$ & $D-\mathrm{H} \cdots A$ \\
\hline $\mathrm{N} 3-\mathrm{H} 2 \mathrm{~N} 3 \cdots \mathrm{N} 1$ & 0.89 & 2.17 & $2.641(14)$ & 112
\end{tabular}


supporting information

$\mathrm{N} 2-\mathrm{H} 1 \mathrm{~N} 2 \cdots \mathrm{S} 1^{\mathrm{i}}$

$\mathrm{N} 3-\mathrm{H} 1 \mathrm{~N} 3 \cdots \mathrm{S} 1^{\mathrm{ii}}$

Symmetry codes: (i) $-x+2,-y+1,-z+2$; (ii) $-x+1,-y,-z+2$.
2.83

2.77
$3.473(11)$

$3.398(11)$
133

130 